\title{
The endocannabinoid system in migraine: from bench to pharmacy and back.
}

\author{
Cristina Tassorelli \\ IRCCS Mondino Foundation; University of Pavia \\ Rosaria Greco \\ IRCCS Mondino Foundation \\ Stephen D. Silberstein \\ Thomas Jefferson University
}

Follow this and additional works at: https://jdc.jefferson.edu/neurologyfp

Part of the Neurology Commons

Let us know how access to this document benefits you

\section{Recommended Citation}

Tassorelli, Cristina; Greco, Rosaria; and Silberstein, Stephen D., "The endocannabinoid system in migraine: from bench to pharmacy and back." (2019). Department of Neurology Faculty Papers. Paper 188.

https://jdc.jefferson.edu/neurologyfp/188

This Article is brought to you for free and open access by the Jefferson Digital Commons. The Jefferson Digital Commons is a service of Thomas Jefferson University's Center for Teaching and Learning (CTL). The Commons is a showcase for Jefferson books and journals, peer-reviewed scholarly publications, unique historical collections from the University archives, and teaching tools. The Jefferson Digital Commons allows researchers and interested readers anywhere in the world to learn about and keep up to date with Jefferson scholarship. This article has been accepted for inclusion in Department of Neurology Faculty Papers by an authorized administrator of the Jefferson Digital Commons. For more information, please contact: JeffersonDigitalCommons@jefferson.edu. 
The endocannabinoid system in migraine: from bench to pharmacy and back

Cristina Tassorelli ${ }^{1,2}$, Rosaria Greco ${ }^{1}$ and Stephen D. Silberstein ${ }^{3}$

${ }^{1}$ Headache Science Center, IRCCS Mondino Foundation, Pavia, Italy

${ }^{2}$ Department of Brain and Behavioral Sciences, University of Pavia, Pavia, Italy

${ }^{3}$ Jefferson Headache Center, Thomas Jefferson University, Philadelphia, PA, USA

\section{Corresponding Author}

Cristina Tassorelli, $\mathrm{MD}, \mathrm{PhD}$

IRCCS C. Mondino Foundation

Via Mondino 2

27100 Pavia (I)

Phone +390382380419

Fsx: +390382380448

e-mail: cristina.tassorelli@unipv.it

\section{Funding:}

No funding was received for this manuscript 


\begin{abstract}
Purpose of the review:

Migraine is a common, highly disabling disorder. Its treatment involves acute and preventive therapy. Many of available preventive medications are not well tolerated, which results in poor compliance and limited effectiveness. Cannabinoids have been proposed for the treatment of migraine but their efficacy and tolerability are controversial.

Recent findings: Cannabinoids modulate functions and activity of signaling pathways that have a key role in pain control. Growing preclinical evidence and initial clinical findings suggest that modulation of the endocannabinoid system, via endogenous or exogenous cannabinoids may be relevant for migraine via multiple mechanisms.
\end{abstract}

Summary: The endocannabinoid system qualifies as an interesting area of research worth exploration in the quest for therapeutic targets for the treatment of migraine.

Key words: endogenous and exogenous cannabinoids, trigeminovascular system, pain 


\section{Introduction}

Our knowledge of the endocannabinoid system (ES) largely originated from studies aimed at identifying the mechanism of action and the properties the plant Cannabis sativa, better known as marijuana. The identification of its main active psychoactive ingredients led to the discovery of the cannabinoid receptors and their endogenous agonists, the endocannabinoids (ECs).

In recent years, much attention has been drawn to the use cannabinoids in several areas of medicine, including pain. It is an area still controversial and debatable that requires careful and focused research.

\section{Cannabinoids}

C. sativa $L$, an annual wind-pollinated dioecious (male and female reproductive organs in separate plants) herb, is one of the world's oldest cultivated plants. $\mathrm{L}$ is an abbreviation for Linnaeus who invented the botanical classification scheme [1]. C. sativa $L$ is usually called "hemp" when used as a source of fiber, "hempseed" when used as a source of seed oil, and "marijuana" (sometimes spelled "marihuana") when used as a drug for therapeutic or recreational purposes. Previously it was believed that there were at least two species; C. sativa L (narrow leaves, branches apart, light green, and tall with few flowers) and Cannabis indica L (wide broad leaves, branches close together, deep green, and short and bushy with dense flowers). Cannabis ruderalis L (varied leaflets and short stature) is sometimes considered a third species.

Many now believe that there is just one species of C. sativa L with subgroups called cultivars. These are selected for a characteristic or combination of characteristics, are distinct, uniform, and stable in these characteristics, and when propagated by appropriate means, retains those characteristics $[2,3]$.

C. sativa $L$ is the species and sativa, indica, and ruderalis are subspecies. Pisupati and colleagues [4] analyzed the nuclear genomic diversity among cannabis varieties, including fiber hemp and seed oil hemp, high cannabinoid drug-types, and feral populations. They found the existence of 
at least three major groups of diversity with European hemp varieties, with genetic groups having different cannabinoid and terpenoid content.

It took more than 100 years to identify the chemical components in cannabis flowers. Over 104 different phytocannabinoids (plant cannabinoids) have been identified including D9tetrahydrocannabinol (THC) and cannabidiol (CBD). Other compounds include terpenes, flavonoids, steroids, noncannabinoid phenols, vitamins, and pigments. These have independent beneficial effects which must be considered when being used therapeutically. CBD and THC are both present in the plant as their carboxylic precursors (D9-tetrahydrocannabinolic acid [THCA] and cannabidiolic acid). They are produced after the heating or drying of the flowers. THCA is synthesized within the glandular trichomes present in the flowers, leaves, and bracts (modified or specialized leaf, associated with a reproductive structure such as a flower) of the female plant. Terpenes are volatile compounds responsible for the typical smell and taste of cannabis. More than 120 different types of terpenes have been identified in cannabis. Terpenes have a wide range of known biological effects. Prior, cannabis classification systems did not take the terpenes into account $[5,6]$.

Today's cannabis plants are hybrid descendants of two genetically divergent gene pools narrow leaf drug (NLD) and broad-leaflet drug (BLD). The original NLD plants had a relatively high THC and low CBD content. BLD later introduced from Afghanistan were short bushy plants with broad, dark green leaflets. BLD plants could produce phytocannabinoids varying from all THC to all CBD. In the late 1970s, growers began to call the original NLD varieties 'sativas' because they resembled narrow leaf hemp fiber varieties. The Afghan BLD hashish varieties were called "indicas." But both NLD and BLD populations belong to different subspecies of $C$. indica, subspecies indica (NLD) and subspecies afghanica (BLD) $[7,8]$.

There is a large disparity between patients, growers, and scientists to describe this plant. Scientists often use the term sativa for low THC content plants and the term "indica" for plants with high THC content. In the "cultural" language "sativa" refers to plants with very high THC content and low or no CBD content whereas indica refers to plants with moderate THC and CBD content. It 
is important to recognize that popular literature about marijuana strains uses the terms indica and sativa in a way that is distinct from the scientific usage and both are inaccurate since form does not determine chemistry[3].

Classifications now take into account the chemical content (chemotype/chemovars) of C. sativa L. Cannabis plants typically exhibit one of the three different chemotypes based on the absolute and relative concentrations of THCA and cannabidiolic acid. Type I refers to high THC chemovars, Type II refers to mixed THC/CBD chemovars, and Type III refers to CBD-predominant chemovars. With modern analytical techniques, rapid and comprehensive analysis of all cannabinoids and terpenes present in cannabis products can be done. This allows an expanded cannabis classification scheme that considers the primary cannabinoid (THC or CBD) and their concentration, terpenoid content and concentration, plant shape, scent, taste, and use.

\section{From cannabis to endocannabinoids}

The search for the site of action of THC led to the discovery of $2 \mathrm{G}$ protein-coupled receptors for THC, named cannabinoid receptor type-1 (CB1) and type-2 (CB2) and the endogenous cannabinoids. endocannabinoids are endogenous lipids that engage cannabinoid receptors. The bestcharacterized endocannabinoids are arachidonoyl ethanolamide, best known as anandamide (AEA) and 2-arachidonoyl glycerol (2-AG). AEA is metabolized mostly by fatty acid amide hydrolase (FAAH), whereas 2-AG by monoglyceride lipase (MAGL) [9]. Precursors of AEA and 2-AG are present in lipid membranes, where they are transformed on demand into the final product and then released into the extracellular space.

Accumulated evidence suggests the existence of additional targets for endocannabinoids in addition to $\mathrm{CB} 1$ and $\mathrm{CB} 2$. This seems the case for the purported ' $\mathrm{CB} 3$ ' receptor GPR55 and the transient receptor potential vanilloid 1 (TRPV1) ion channel. Other potential endocannabinoid targets, such as peroxisome proliferator-activated receptor a and g are localized in the nucleus, where they shuttle from/to the cytosol in a ligand-dependent manner. 
$\mathrm{CB}$ receptors, endogenous ligands that activate them, and the enzymes responsible for the synthesis and degradation of the endocannabinoids constitute altogether the endocannabinoid system.

AEA is responsible for maintaining basal endocannabinoid tone and has a high selectivity for the $\mathrm{CB} 1$ receptor over the peripheral $\mathrm{CB} 2$ receptor [9]. AEA also binds to the TRPV1, an ionotropic receptor that is responsible for the integration of noxious stimuli that cause pain [10]. In contrast to AEA, 2-AG is a full agonist for both $\mathrm{CB} 1$ and $\mathrm{CB} 2$ [9]. $\mathrm{CB} 1$ receptors are found in neuroanatomical regions involved in pain processing and modulate the release of several neurotransmitters [11]. They are also expressed in afferent fibers and in many nonneural cells. CB1 receptors are involved in pain transmission and modulation at multiple levels of the neuroaxis from periphery to central nervous system $[9,12]$. CB2 receptors are located primarily in immune cells, even though CB2 mRNA was detected within the spinal cord and CB2 protein in the brain [13]. CB receptors co-localize with opioid receptors and augment the analgesic effects of opioids, probably via pharmacodynamics mechanisms. GPR55 is located in the brain, in the peripheral nervous system and in mast cells [14]. The enzymes involved in the synthesis of AEA and 2-AG are the Nacylphosphatidylethanolaminephospholipase D and the sn-1-specific diacylglycerol lipase, respectively [9]. Once synthesized and released, endocannabinoids are removed from the extracellular space through an endocannabinoid membrane transporter (EMT) and subsequently hydrolyzed. AEA is mostly hydrolyzed by FAAH, which releases, arachidonic acid and ethanolamine or arachidonic acid and glycerol, whereas 2-AG degradation is mainly because of a cytosolic MAGL [9].

The theoretical bases for a role of the endocannabinoid system in migraine pain: Cannabinoids and Endocannabinoids

THC, the constituent responsible for the mind-altering and intoxicating effects of C. Sativa, acts on $\mathrm{CB} 1$ and $\mathrm{CB} 2$ receptors. $\mathrm{CBD}$ binds to other receptors, and is devoid of the psychoactive effects associated with THC. CBD is the most abundant cannabis-derived non-CB1/CB2 receptor ligand, that may exert some effects via inhibition of FAAH $[15,16]$. In addition to its psychotropic properties, 
cannabis has long been known to have analgesic, immunomodulatory, and anti-inflammatory effects [17]. CBD reduces inflammatory and neuropathic pain by modulation of $5-\mathrm{HT} 1_{\mathrm{A}}$ (serotonin $1 \mathrm{~A}$ ) receptors and TRPV1 channels [18\&]. Similarly, to some other cannabinoids, CBD suppresses cytokines and chemokines release, decreases production of reactive oxygen species and modulates immune cell system [19] (Table 1).

Experimental studies show that THC is effective in reducing multiple types of pain including pain caused by acute noxious stimuli and chronic inflammation [43]. THC prevents depression of home cage wheel running caused by migraine-like pain after dural TRPA1 agonist microinjection, in a timeand dose-dependent manner [44,45]. Moreover, THC and other CB1 agonists dose-dependently reduce cortical spreading depression amplitude, duration, and propagation velocity in a rat model [26]. WIN 55,212-2, a potent CB1 agonist, inhibits trigeminocervical complex A and C-fiber afferent activity [46].

Theoretically, the endocannabinoid system may target migraine in multiple pathways (glutamatergic, serotoninergic, opiatergic and inflammatory) [47-49] and at multiple anatomic levels. In the periphery, endocannabinoids may affect neurogenic inflammation mediated by the trigeminovascular system in the meninges via their stabilizing effect on mast cells $[14,50]$ and the inhibition of calcitonin gene-related peptide (CGRP)-induced dilatation of dural blood vessels and neuronal pronociceptive activity [51]. Centrally, activation of CB1 receptors in the ventrolateral periaqueductal gray (PAG) modulates nociceptive trigeminovascular transmission in the trigeminocervical complex via a mechanism mediated by 5HT1B/1D receptors [52]. Endocannabinoid system also affects the descending pathways of pain control by acting at either CB1 or TRPV1 receptors $[53,54]$. Consistently, microinjections within the PAG and rostral ventrolateral medulla of URB597 (cyclohexylcarbamic acid 3'-carbamoylbiphenyl-3-yl ester), a global FAAH inhibitor, enhance analgesia via TRPV1 and CB1 receptors in animal models of neuropathic pain [53]. Again, the levels of AEA and 2-AG are significantly increased in animal models of neuropathic pain, to suggest a compensatory upregulation of endocannabinoids directed at the inhibition of pain 
in pathological conditions [55]. AEA acts presynaptically to prevent release of nitric oxide by CGRP in the dura mater [51]. Also, artificially increased levels of 2-AG and AEA in the dorsal PAG enhance stress induced analgesia [56].

Studies conductedonthemigraine-specific animal model based on nitroglycerin (NTG) administration showed increased FAAH activity and up-regulation of CB1 receptor binding sites in the rat hypothalamus and in the medulla [57]. In NTG-treated rats, activity of FAAH and MAGL hydrolases increased in the mesencephalon, a key area in migraine pathophysiology [57], to suggest a decrease in the endocannabinoid tone. Consistently, we observed that AEA administration significantly reduced NTG-induced nocifensive behavioural and neuronal activation in the nucleus trigeminal caudalis (NTC), another key area for migraine [58], and others reported reduced central sensitization through TRPV1, cyclooxygenase-2 expression, and nuclear factor kappa B inhibition in NTC [59]. If AEA and CB1-mediated mechanisms are important, we must not overlook the possible role of CB2-mediated mechanisms when considering that the activation of these receptors significantly decreases nocifensive behaviour in rats previously made hyperalgesic by NTG [60]. Modulation of CB2 receptor activity seems particularly appealing, as they induce analgesic effects without producing tolerance or central side-effects $[61,62]$.

FAAH inhibition causes analgesia and reduces inflammation in animal models of migraine pain $[63,64]$. NTG-induced mechanical allodynia and c-Fos protein in the NTC are both suppressed in FAAH deficient mice or after URB597 treatment, thus strongly indicating a role for AEA in migraine pain [64]. In agreement, URB937, a peripherally restricted FAAH inhibitor, reduces NTG-induced nocifensive behavior, and c-Fos expression in the NTC and locus coeruleus [63], thus underscoring the role of peripheral mechanisms, possibly related to the anti-inflammatory activity of endocannabinoids [65\&\&]. Indeed, URB937 acts only peripherally, maintaining higher levels of AEA released by nervous terminal located in the injured peripheral tissues or in the dura, via CB1 receptor activation in trigeminovascular endings [66]. It is worth noting that similar effects have been reported with biphenyl3-ylcarbamic acid cyclohexyl ester (URB602), a reversible MAGL inhibitor that significantly 
decreases NTG induced neuronal activation in PAG and NTC, to suggest once more that modulation of CB2 may also play a role in migraine [67\&\&]. These findings support the hypothesis that modulation of the endocannabinoid system may be an extremely valuable approach for the treatment of migraine-related pain and hyperalgesia [68]. However, controversy exists as regards the safety of the modulation of the endocannabinoid system, in particular as regards the inhibition of endocannabinoids catabolism when off-target effect is not properly ruled out [69]. Technological advances allow a full characterization of protein interaction landscape and warrants further investigation along this promising pathway.

\section{Clinical data}

Clinical evidence in favor of the need to further investigate the role of the endocannabinoid system in migraine is more scattered, but nonetheless compelling. Women with migraine without aura bear increased activities of FAAH and EMT, to suggest reduced AEA levels [70]. In another study based on PET detection of $\mathrm{CB} 1$ receptors, women with episodic migraine showed increased binding in painmodulating areas of the brain [71]. Variants in the CB1 receptor gene increase the risk of migraine attack with nausea in life stress exposed patients [72\&]. A more recent study failed to detect significant changes in the plasma levels of AEA and other fatty acid ethanolamides in episodic migraineurs [73], probably as a consequence of a high interpatient variability in the evaluated cohorts.

A more consistent alteration of endocannabinoid system seems to be implicated in chronic migraine. FAAH and EMT levels were lower in the platelets of patients with chronic migraine when compared to either controls or episodic migraine [74]. Furthermore, FAAH levels decreased in patients with chronic migraine and acute medication overuse after detoxification, in parallel with the clinical improvement and with the restored pain control, to suggest that the catabolizing pathway is altered in these patients [75]. Increased levels of N-palmitoylethanolamine (PEA), a fatty acid amide belonging to the endocannabinoid system, were found in the cerebrospinal fluid of chronic migraine patients [76], a finding interpreted by the authors as a compensatory mechanism.

\section{Cannabinoids in the management of migraine: Clinical effects and Tolerability}


PEA has showed properties that may be useful in pain conditions [77,78]. Limited evidence suggests the possible efficacy of ultramicronized PEA in reducing the number of migraine attacks/month, regardless of age or sex, and the use of acute medications $[79,80]$.

Cannabis-based medicines are approved only for a few indications. None are approved for pain or headache. The Health Effects of Cannabis and Cannabinoid report [81] concluded that there is substantial evidence that cannabinoids are effective for the treatment chronic pain in adults. But little is known about the efficacy, dose, routes of administration, or side effects of available cannabis. Anecdotal evidence suggests a role for cannabis in the treatment of headache and migraine [82,83], but no controlled clinical studies have been done. Anecdotal benefit has been reported in pseudotumor cerebri [84] and refractory cluster headaches [85]. Cannabis use is very frequent in French cluster headache patients; most had variable, uncertain, or even negative effects of cannabis smoking [86]. Headache is an adverse event associated with cannabinoid medications, and is very common with cannabis withdrawal [87]. It is therefore possible that using cannabis simply relieves headache caused by cannabis withdrawal.

Rhyne et al. [88] did a retrospective chart review of 121 adults with migraine headache referred for treatment with medical marijuana and had at least one follow-up visit. Migraine headache frequency decreased from 10.4 to 4.6 headaches per month $(P<0.0001)$ and self-reported positive effects were recorded in 48 patients (39.7\%). The scientific validity of this study is however limited by multiple issues: high rate of drop-outs, inconsistent chart documentation, and so on.

Nabilone is a synthetic cannabinoid with a highly selective agonistic activity for CB1 and CB2 receptors. It decreased analgesic intake and improved pain in a small double-blind, placebocontrolled trial of 26 patients with treatment refractory medication overuse headache, a frequent complication of chronic migraine [89]. They were given nabilone $(0.5 \mathrm{mg})$ or ibuprofen (400 $\mathrm{mg})$ for 8 weeks, then after a week-long washout period, switched to the other drug for a second 8-week course. Nabilone was significantly more effective than ibuprofen in reducing pain intensity, analgesic intake, as well as in improving quality of life. Patients only had mild adverse effects. A different 
multicenter, double-blind, placebo-controlled study of the safety and efficacy of a dronabinol, a synthetic form of $\mathrm{THC}$, delivered with a metered dose inhaler for the treatment of migraine (clincaltrials.gov, The national clinical trial (NCT) Identifier: NCT00123201) has been completed for several years, without publication of results from the sponsor Solvay Pharmaceuticals, Brussels, Belgium.

Tolerability is a concern in the use of cannabinoids. Dosing is individualized and requires titration. Those with no prior experience should start with a very low dose and stop if undesirable side-effects occur. Psychoactive effects occur at doses above the individual consumer's psychotropic threshold. They are generally pleasurable and relaxing. However, this can turn into dysphoria, anxiety, or even panic. Impairment of memory, reductions in psychomotor and cognitive performance, and disordered perception of the passage of time can occur. Common physical effects are tiredness, dizziness, tachycardia, orthostatic hypotension, dry mouth, reduced lacrimation, muscle relaxation, and increased appetite. Tolerance develops too many of these undesired effects of cannabinoids particularly tiredness, dizziness, and cardiovascular and psychoactive effects over a period of days or weeks [90].

There is an association between cannabis use and the development of psychosis, at least in vulnerable subjects [91], with the highest risk among the most frequent users. The evidence is unclear as to whether and how cannabis use is associated with heart attack, stroke, and diabetes, but tachycardia often occurs with acute dosage; tolerance developing with chronic exposure. Cannabis use increases the risk of being involved in a motor vehicle accident. Smoking cannabis during pregnancy is linked to lower birth weight in the offspring. The relationship between smoking cannabis during pregnancy and other pregnancy and childhood outcomes is unclear. The hyperemesis syndrome can occur in chronic daily cannabis users. It is characterized by severe, intractable episodes of nausea and cyclic vomiting accompanied by abdominal pain (typically epigastric or periumbilical); relieved by compulsive hot water bathing or showering [92]. Treatment includes: cessation of cannabis use, rehydration, and psychological counseling. 


\section{Conclusions}

C. sativa is one of the world's oldest cultivated plants. It has been used for thousands of years. Many believe it is effective for migraine and headache, but controlled clinical trials are missing. Anecdotal information does not provide THC and CBD content or the amount and type of terpenes. Cannabis is effective for pain, we still need to know if it is effective for headache.

The modulation of the endocannabinoid system, in particular in the processing of nociceptive signals in the trigeminovascular system, may prove a well-tolerated and pharmacologically sound therapeutic option for migraine [93]. Additional studies are needed to explore the neurobiological mechanisms and neural circuits involved.

\section{KEY POINTS}

1) Alterations in the Endocannabinoid System seem to be implicated in migraine pathogenesis and progression

2) Exogenous and endogenous cannabinoids may have therapeutic benefits in migraine, provided that tolerability issues are addressed

3) More research is necessary for the complete characterization of the multiple components of Cannabis and for the identification of safe modulators of the endogenous cannabinoid system as suitable targets for migraine treatment.

\section{Acknowledgements}

We are grateful to Prof. Daniele Piomelli for providing well characterized multiple inhibitors of the degrading enzymes of endocannabinoids for our experiments in animal models of migraine.

\section{Financial support and sponsorship}

None

\section{Conflicts of interest}


Cristina Tassorelli has received honoraria for participation in clinical trials, contribution to advisory boards or scientific presentations from: Allergan, Electrocore, Eli-Lilly, Novartis, Teva and Medscape, LLC. CT has received independent support from the Italian Ministry of Health, Italian Ministry of Research and European Community. CT has no ownership interest and does not own stocks of any pharmaceutical company. CT serves as Chief Section Editor of Frontiers in Neurology - Section Headache Medicine and Facial Pain and on the editorial board of Journal of Headache and Pain. CT is the chairman of the Clinical Trials Committee of the IHS.

$\underline{\text { Rosaria Greco has no conflict of interest to declare. }}$

Stephen Silberstein is the Director of the Jefferson Headache Center in Philadelphia, PA. As a consultant and/or advisory panel member, he receives, or has received, honoraria from Abide Therapeutics; Alder Biopharmaceuticals; Allergan, Inc.; Amgen; Avanir Pharmaceuticals, Inc.; Biohaven Pharmaceuticals; Cefaly; Curelator, Inc.; Dr. Reddy's Laboratories; Egalet Corporation; GlaxoSmithKline Consumer Health Holdings, LLC.; eNeura Inc.; electroCore Medical, LLC; Impel NeuroPharma, Inc.; Lilly USA, LLC; Medscape, LLC; Novartis, Inc.; Satsuma Pharmaceuticals; Supernus Pharmaceuticals, Inc.; Teva Pharmaceuticals; Theranica; and Trigemina, Inc.

The Authors have no conflicts of interest to declare as regards the substances mentioned in this

manuscript.

\section{References}

1. Russo EB. History of cannabis and its preparations in saga, science, and sobriquet. Chemistry and Biodiversity. 2007; 4:1614-1648.

2. McNeill JFR, Barrie WR, Buck V, et al. International Code of Nomenclature for Algae, Fungi, and Plants. Wiersema \& N. J. Turland. (eds.). 2012.

3. Small E. Evolution and Classification of Cannabis sativa (Marijuana, Hemp) in Relation to Human Utilization Bot. Rev. 2015; 81:189-294.

4. Pisupati R, Vergara D, Kane NC. Diversity and evolution of the repetitive genomic content in Cannabis sativa. BMC Genomics 2018;19(1):156.

5. Lu HC, Mackie K. An Introduction to the Endogenous Cannabinoid System. Biological Psychiatry 2016; 79:516-525.

6. Multiple authors. The Health Effects of Cannabis and Cannabinoids: The Current State of Evidence and Recommendations for Research, The National Academy of Science, 2017.

7. Clarke RC, Merlin MD. Cannabis Domestication, Breeding History, Present-day Genetic Diversity, and Future Prospects. Critical Reviews in Plant Sciences 2016;35,5-6:293-327.

8. Lynch RC, Vergara D, Tittes S, White K, Schwartz CJ, Gibbs MJ, Ruthenburg TC, deCesare K, Land DP, Kane NC. Genomic and Chemical Diversity in Cannabis, Critical Reviews in Plant Sciences 2016;35,5-6:349-363,7

9. Zou S, Kumar U. Cannabinoid Receptors and the Endocannabinoid System: Signaling and Function in the Central Nervous System. Int. J. Mol. Sci. 2018; 13:19.

10. Starowicz K, Makuch W, Osikowicz M, et al. Spinal anandamide produces analgesia in neuropathic rats: possible $\mathrm{CB}(1)$ - and TRPV1-mediated mechanisms. Neuropharmacology 2012; 62:1746-1755.

11. Woodhams SG, Sagar DR, Burston JJ, Chapman V. The role of the endocannabinoid system in pain. Handb. Exp. Pharmacol. 2015;119-143.

12. Kendall DA, Yudowski GA. Cannabinoid Receptors in the Central Nervous System: Their Signaling and Roles in Disease. Front. Cell Neurosci. 2017;10:294. 
13. Zhang HY, Shen H, Jordan CJ, Liu QR, Gardner EL, Bonci A, Xi ZX. CB(2) receptor antibody signal specificity: correlations with the use of partial $\mathrm{CB}(2)$-knockout mice and anti-rat $\mathrm{CB}(2)$ receptor antibodies. Acta Pharmacol Sin. 2018.

14. Cantarella G, Scollo M, Lempereur L, et al. Endocannabinoids inhibit release of nerve growth factor by inflammation-activated mast cells. Biochem. Pharmacol. 2011;82:380-388.

15. Leweke FM, Piomelli D, Pahlisch F, et al. Cannabidiol enhances anandamide signaling and alleviates psychotic symptoms of schizophrenia. Transl. Psychiatry 2012;2:e94.

16. Leishman E, Manchanda M, Thelen R, et al. Cannabidiol's Upregulation of N-acyl Ethanolamines in the Central Nervous System Requires N-acyl Phosphatidyl EthanolamineSpecific Phospholipase D. Cannabis Cannabinoid Res. 2018 Nov 30;3:228-241.

17. Noreen N, Muhammad F, Akhtar B, Azam F, Anwar MI. Is Cannabidiol a Promising Substance for New Drug Development? A Review of its Potential Therapeutic Applications. Crit. Rev. Eukaryot. Gene Expr. 2018;28:73-86.

18. De Gregorio D, McLaughlin RJ, Posa L, et al. Cannabidiol modulates serotonergic transmission and reverses both allodynia and anxiety-like behavior in a model of neuropathic pain. Pain 2019 160:136-150.

*The Authors show that repeated treatment with low-dose CBD blocks sensitization in an animal model of neuropathic pain via TRPV1 activation, reduces anxiety through 5-HT1A receptor activation, and rescues impaired 5-HT neurotransmission.

19. Booz GW. Cannabidiol as an emergent therapeutic strategy for lessening the impact of inflammation on oxidative stress. Free Radic. Biol. Med. 2011; 51:1054-1061.

20. Russo EB. Cannabis Therapeutics and the Future of Neurology. Front. Integr. Neurosci. 2018; 18:12:51.

21. Kandasamy R, Dawson CT, Craft RM, Morgan MM. Anti-migraine effect of $\Delta(9)$ tetrahydrocannabinol in the female rat. Eur. J. Pharmacol. 2018; 818:271-277.

22. Kandasamy R, Dawson CT, Hilgendorf TN, Morgan MM. Medication overuse headache following repeated morphine, but not [INCREMENT]9-tetrahydrocannabinol administration in the female rat. Behav Pharmacol. 2018;29:469-472.

23. Kazemi H, Rahgozar M, Speckmann EJ, et al. Effect of cannabinoid receptor activation on spreading depression. Iran. J. Basic Med. Sci. 2012;15:926-936.

24. Akerman S, Holland PR, Goadsby PJ. Cannabinoid (CB1) receptor activation inhibits trigeminovascular neurons. J. Pharmacol. Exp. Ther .2007; 320: 64-71.

25. Greco R, Demartini C, Zanaboni AM, et al. Endocannabinoid System and Migraine Pain: An Update. Front. Neurosci. 2018 19;12:172.

26. Mendiguren A, Aostri E, Pineda J. Regulation of noradrenergic and serotonergic systems by cannabinoids: relevance to cannabinoid-induced effects. Life Sci. 2018; 192:115-127.

27. Zubrzycki M, Janecka A, Liebold A, et al. Effects of centrally administered endocannabinoids and opioids on orofacial pain perception in rats. Br. J. Pharmacol. 2017;174:3780-3789.

28. Sugawara K, Bíró T, Tsuruta D, et al. Endocannabinoids limit excessive mast cell maturation and activation in human skin. J Allergy Clin Immunol. 2012; 129:726-738.

29. Akerman S, Kaube H, Goadsby PJ. Anandamide is able to inhibit trigeminal neurons using an in vivo model of trigeminovascular-mediated nociception. J Pharmacol Exp Ther. 2004; 309:56-63.

30. Akerman S, Holland PR, Lasalandra MP, Goadsby PJ. Endocannabinoids in the brainstem modulate dural trigeminovascular nociceptive traffic via $\mathrm{CB} 1$ and "triptan" receptors: implications in migraine. J Neurosci. 2013 11;33:14869-14877.

31. Maione S, Bisogno $\mathrm{T}$, de Novellis et al. Elevation of endocannabinoid levels in the ventrolateral periaqueductal grey through inhibition of fatty acid amide hydrolase affects descending nociceptive pathways via both cannabinoid receptor type 1 and transient receptor potential vanilloid type-1 receptors. J Pharmacol Exp Ther. 2006; 316:969-982. 
32. Burston JJ, Woodhams SG. Endocannabinoid system and pain: an introduction. Proc Nutr Soc. 2014 Feb;73(1):106-17

33. Petrosino S, Palazzo E, de Novellis V, et al. Changes in spinal and supraspinal endocannabinoid levels in neuropathic rats. Neuropharmacology 2007; 52: 415-422.

34. Hohmann AG, Suplita RL, Bolton NM, et al. An endocannabinoid mechanism for stressinduced analgesia. Nature 2005; 23;435(7045):1108-1112.

35. Greco R, Gasperi V, Sandrini G, et al. Alterations of the endocannabinoid system in an animal model of migraine: evaluation in cerebral areas of rat. Cephalalgia 2010;30:296-302.

36. Greco R, Mangione AS, Sandrini G, et al. Effects of anandamide in migraine: data from an animal model. J Headache Pain 2011;12:177-183.

37. Nagy-Grócz G, Tar L, Bohár Z, et al. The modulatory effect of anandamide on nitroglycerininduced sensitization in the trigeminal system of the rat. Cephalalgia 2016;36:849-861.

38. Greco R, Mangione AS, Sandrini G, Nappi G, Tassorelli C. Activation of CB2 receptors as a potential therapeutic target for migraine: evaluation in an animal model. J Headache Pain.2014; 15:14.

39. Romero TR, Resende LC, Guzzo LS, Duarte ID. CB1 and CB2 cannabinoid receptor agonists induce peripheral antinociception by activation of the endogenous noradrenergic system. Anesth. Analg. 2013;116: 463-472.

40. Vincenzi F, Targa M, Corciulo C, et al. Antinociceptive effects of the selective CB2 agonist MT178 in inflammatory and chronic rodent pain models. Pain 2013, 154:864-873.

41. Greco R, Bandiera T, Mangione AS, Demartini C, et al. Effects of peripheral FAAH blockade on NTG-induced hyperalgesia-evaluation of URB937 in an animal model of migraine. Cephalalgia 2015; 35:1065-1076.

42. Nozaki C, Markert A., Zimmer A. Inhibition of FAAH reduces nitroglycerin-induced migraine-like pain and trigeminal neuronal hyperactivity in mice. Eur. Neuropsychopharmacol. 2015; 25:1388-1396.

43. van Niekerk G, Mabin T, Engelbrecht AM. Anti-inflammatory mechanisms of cannabinoids: an immunometabolic perspective. Inflammopharmacology 2019. doi: 10.1007/s10787-01800560-7. [Epub ahead of print].

** A thorough review of the AMPK-mediated mechanisms in the anti-inflammatory effect of cannabinoids.

44. Agarwal N, Pacher P, Tegeder I, et al. Cannabinoids mediate analgesia largely via peripheral type 1 cannabinoid receptors in nociceptors. Nat Neurosci. 2007;10:870-879.

45. Greco R, Demartini C, Zanaboni A M, et al. Inhibition of monoacylglycerol lipase: another signalling pathway for potential therapeutic targets in migraine?. Cephalalgia 2018; 38:11381147.

**The findings show that inhibition of monoacylglycerol lipase, which inhibits 2-AG degradation, reduces in vivo nitroglycerin-induced hyperalgesia in the trigeminal region.

46. Maione S, Costa B, Di Marzo V. Endocannabinoids: a unique opportunity to develop multitarget analgesics. Pain 2013; 154: S87-S93.

47. van Esbroeck ACM, Janssen APA, Cognetta AB et al. Activity-based protein profiling reveals off-target proteins of the FAAH inhibitor BIA 10-2474. Science 2017;356:1084-1087.

48. Cupini L M, Bari M, Battista N, et al. Biochemical changes in endocannabinoid system are expressed in platelets of female but not male migraineurs. Cephalalgia 2006; 26:277-281.

49. Van der Schueren BJ, Van Laere K, Gérard N, et al. Interictal type 1 cannabinoid receptor binding is increased in female migraine patients. Headache 2012;52:433-440.

50. Juhasz G, Csepany E, Magyar M, et al. Variants in the CNR1 gene predispose to headache with nausea in the presence of life stress. Genes Brain Behav. 2017;16:384-393.

*The paper shows that also genetic variants in human CB1 receptor gene may increase the risk of headache 
51. Gouveia-Figueira S, Goldin K, Hashemian SA, et al. Plasma levels of the endocannabinoid anandamide, related $\mathrm{N}$-acylethanolamines and linoleic acid-derived oxylipins in patients with migraine. Prostaglandins Leukot. Essent. Fatty Acids 2017; 120:15-24.

52. Cupini LM, Costa C, Sarchielli P, et al. Degradation of endocannabinoids in chronic migraine and medication overuse headache. Neurobiol. Dis. 2008; 30:186-189.

53. Perrotta A, Arce-Leal N, Tassorelli C et al. Acute reduction of anandamide-hydrolase (FAAH) activity is coupled with a reduction of nociceptive pathways facilitation in medication-overuse headache subjects after withdrawal treatment. Headache 2012; 52: 1350-1361.

54. Sarchielli P, Pini LA, Coppola F, et al. Endocannabinoids in chronic migraine: CSF findings suggest a system failure. Neuropsychopharmacology 2007;32:1384-1390.

55. Boccella S, Cristiano C, Romano R, et al. Ultra-micronized palmitoylethanolamide rescues the cognitive decline-associated loss of neural plasticity in the neuropathic mouse entorhinal cortex-dentate gyrus pathway. Neurobiol Dis. 2019;121:106-119.

56. Passavanti MB, Alfieri A, Pace MC, et al. Clinical applications of palmitoylethanolamide in pain management: protocol for a scoping review. Syst Rev. 2019; 8:9.

57. Chirchiglia D, Cione E, Caroleo MC, et al. Effects of Add-On Ultramicronized N-Palmitol Ethanol Amide in Patients Suffering from Migraine With Aura: A Pilot Study. Front Neurol. 2018; 19:674.

58. Dalla Volta G, Zavarize P, Ngonga FK Gaelle, Carli D. Ultramicronized Palmitoylethanolamide Reduces Frequency and Pain Intensity in Migraine. A Pilot Study. Int. J. Neurol. Brain Disord. 2016; 1:13-17.

59. Multiple Authors. The Health Effects of Cannabis and Cannabinoids National Academies Press, Washington (DC): 2017. pp:1-486, https://doi.org/10.17226/24625

60. Borgelt LM, Franson KL, Nussbaum AM, Wang GS Review The pharmacologic and clinical effects of medical cannabis.Pharmacotherapy. 2013; 33:195-209.

61. Baron EP. Medicinal Properties of Cannabinoids, Terpenes, and Flavonoids in Cannabis, and Benefits in Migraine, Headache, and Pain: An Update on Current Evidence and Cannabis Science. Headache 2018 Jul;58(7):1139-1186.

62. Evans RW, Ramadan NM. Are cannabis-based chemicals helpful in headache? Headache 2004;44:726-727.

63. Robbins, MS., Tarshish S, Solomon S et al. Cluster attacks responsive to recreational cannabis and dronabinol. Headache 2009; 49: 914-916.

64. Leroux E, Taifas I, Valade D et al. Use of cannabis among 139 cluster headache sufferers. Cephalalgia. 2012; 33: 208-213.

65. Levin KH, Copersino, ML, Heishman, SJ, et al. Cannabis withdrawal symptoms in nontreatment-seeking adult cannabis smokers. Drug Alcohol Depend. 2012; 111: 120-127.

66. Rhyne DN, Anderson SL, Gedde M, Borgelt LM. Effects of Medical Marijuana on Migraine Headache Frequency in an Adult Population. Pharmacotherapy. 2016;36:505-510.

67. Pini LA, Guerzoni S, Cainazzo MM, et al. Nabilone for the treatment of medication overuse headache: results of a preliminary doubleblind, active-controlled, randomized trial. J Headache Pain. 2012;13: 677-684.

68. Grotenhermen F, Muller-Vahl K. Medicinal Uses of Marijuana and Cannabinoids Critical Reviews in Plant Sciences. 2016; 35:378-405.

69. Ortiz-Medina MB, Perea M, Torales J, Ventriglio A, Vitrani G, Aguilar L, Roncero C. Cannabis consumption and psychosis or schizophrenia development. Int J Soc Psychiatry. 2018;64:690-704.

70. Simonetto, DA, Oxentenko AS, Herman ML et al. Cannabinoid hyperemesis: a case series of 98 patients. Mayo Clin.Proc. 2012; 87:114-119.

71. Piomelli D, Sasso O. Peripheral gating of pain signals by endogenous lipid mediators. Nat. Neurosci 2014;17:164-174. 
